\title{
Cancer risk and management in a woman with Peutz-Jeghers syndrome
}

\author{
Koji Yasumoto $\cdot$ Ayako Suzuki $\cdot$ Noriomi Matsumura $\cdot$ Tsukasa Baba $\cdot$ \\ Yoko Oiwa - Shin'ichi Miyamoto $\cdot$ Minoru Matsuura $\cdot$ Kazutaka Obama \\ Aki Kido · Shigeaki Umeoka - Sachiko Minamiguchi - Yoshiki Mikami · \\ Yumiko Yoshioka $\cdot$ Kenzo Kosaka $\cdot$ Masaki Mandai $\cdot$ Ikuo Konishi
}

Received: 20 December 2011/ Accepted: 24 January 2012/Published online: 29 February 2012

(C) The Japan Society of Clinical Oncology 2012

\begin{abstract}
Peutz-Jeghers syndrome (PJS) is known to cause an elevated risk of cancer development. We present the case of a 41-year-old Japanese woman with PJS who had various lesions in the abdominal and pelvic organs. The preoperative diagnosis was a right ovarian tumor suspected to be a mucinous borderline tumor, a uterine cervical lesion suspected to be lobular endocervical glandular hyperplasia (LEGH), gastrointestinal hamartomatous polyps, and intestinal invagination. She underwent hysterectomy, right salpingo-oophorectomy, left salpingectomy, and partial resection of the jejunum. The pathological diagnosis was an endometriotic cyst of the right ovary, atypical LEGH of the
\end{abstract}

K. Yasumoto $\cdot$ A. Suzuki $(\bowtie) \cdot$ N. Matsumura $\cdot$ T. Baba Y. Oiwa · Y. Yoshioka $\cdot$ K. Kosaka $\cdot$ M. Mandai · I. Konishi Department of Gynecology and Obstetrics, Kyoto University Hospital and Graduate School of Medicine, 54 Shogoin Kawahara-cho, Sakyo-ku, Kyoto 606-8507, Japan

e-mail: flapper@kuhp.kyoto-u.ac.jp

S. Miyamoto · M. Matsuura

Department of Gastroenterology and Hepatology, Kyoto University Hospital and Graduate School of Medicine, 54 Shogoin Kawahara-cho, Sakyo-ku, Kyoto 606-8507, Japan

K. Obama

Department of Gastrointestinal Surgery, Kyoto University Hospital and Graduate School of Medicine,

54 Shogoin Kawahara-cho, Sakyo-ku, Kyoto 606-8507, Japan

A. Kido $\cdot$ S. Umeoka

Department of Diagnostic Imaging and Nuclear Medicine, Kyoto University Hospital and Graduate School of Medicine, 54

Shogoin Kawahara-cho, Sakyo-ku, Kyoto 606-8507, Japan

S. Minamiguchi · Y. Mikami

Department of Diagnostic Pathology, Kyoto University Hospital and Graduate School of Medicine, 54 Shogoin Kawahara-cho, Sakyo-ku, Kyoto 606-8507, Japan cervix, gastric metaplasia of the fallopian tube, intestinal hamartomatous polyps, and adenocarcinoma in a polyp of the jejunum. This case is representative for women with PJS who may develop multifocal malignancies positively based on the germline mutation of the LKB1/STK11 gene. The cancer risk, surveillance guidelines, and management for women with PJS were discussed in this case conference.

Keywords Peutz-Jeghers syndrome (PJS) - Cancer risk · Hamartomatous polyps · Intestinal adenocarcinoma . Ovarian tumor $\cdot$ Lobular endocervical glandular hyperplasia (LEGH) · Gastric metaplasia

\section{Case presentation}

Dr. Suzuki (Gynecologist, Chairperson of the conference) Good afternoon! Today, we would like to review the cancer risk, surveillance, and management in women with PeutzJeghers syndrome (PJS). First, we will have a presentation of a patient with PJS having various lesions, and then discuss the clinical assessment, treatment, and specific pathology.

Drs. Yasumoto and Oiwa (Gynecologists) The patient is a 41-year-old, nulligravid woman, with a desire for further examination of her abdominal mass. Family history revealed that her mother and sister had been diagnosed with PJS (Fig. 1). In addition, the mother had both breast and duodenal cancers, and died of the latter malignancy at the age of 48. The elder sister also died from uterine cervical cancer at the age of 30 .

The past history and present illness revealed that she had an emergent laparotomy at the age of 10 for intestinal intussusception. At that time, she was diagnosed with PJS 


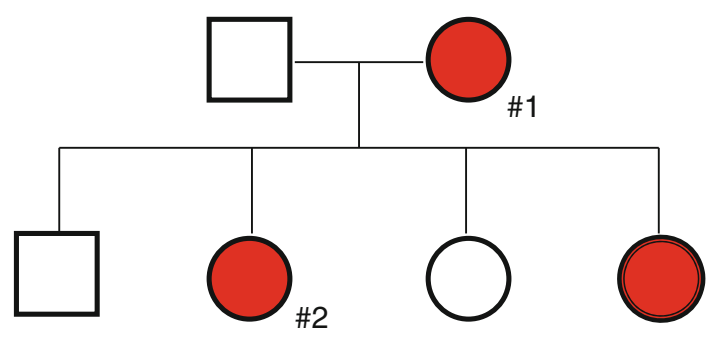

Fig. 1 Family tree. The patient is the youngest child of four siblings. The mother (no. 1) and the second older sister (no. 2) were diagnosed with PJS. It is unclear whether the grandfather and grandmother also had PJS

based on her family history, lip pigmentation, and intestinal polyps. She received annual endoscopic examinations while between 10 and 26 years of age. At the age of 20, she again underwent laparotomy because of intestinal obstruction. However, she discontinued the examinations after 26 years of age. Her menstrual history showed that menarche occurred at the age of 12 , and she had regular cycles of 28-day intervals with mild dysmenorrhea. She had no history of pregnancy.

When the patient was 40 years of age, the health screening revealed a lower abdominal tumor, anemia, and positivity for fecal occult blood. She then underwent computed tomography (CT) of the abdomen, which showed a tumor in the left upper quadrant, a large pelvic mass, and a uterine cervical lesion. She was referred to our hospital for further examination and hospitalized for treatment.

On admission, physical examination showed pigmentation of the lips and hands, and a small nodule in the right $\mathrm{CD}$ region of the breast. Her abdomen was diffusely swollen, and there were tumors with poor mobility. Gynecological examination revealed that the uterine cervix was enlarged. On bimanual palpation, on the right side of the uterus, there was a tense and elastic tumor approximately $15 \mathrm{~cm}$ in diameter. Transabdominal ultrasonography (US) revealed the tumor to be a huge, multilocular cystic tumor with a part having a thick wall with small solid protrusion. The serum tumor maker CA125 level was elevated to $587 \mathrm{IU} / \mathrm{ml}$ (normal range $<35 \mathrm{IU} / \mathrm{ml}$ ). All of these findings suggested the possibility of ovarian mucinous borderline or malignant tumor.

Transvaginal US also showed the presence of multiple cystic lesions in the cervix. Cervical cytology showed no malignancy, but suggested the existence of endocervical glandular lesions of gastric phenotype. Her cervical mucus was positive for the latex agglutination test using the HIK1083 antibody against gastric mucin. These findings strongly suggested the presence of lobular endocervical glandular hyperplasia (LEGH) of the uterine cervix, rather than that of minimal deviation adenocarcinoma (MDA).
Dr. Kido (Radiologist) On MRI images of the pelvis (Fig. 2), there was a $22 \times 16-\mathrm{cm}$ bilocular cystic tumor with a distinct boundary (Fig. $2 a-c$ ). Since the left ovary could be detected on the left dorsal to the bladder and appeared normal, the tumor was thought to have originated from the right ovary (Fig. 2d). On both T1- and T2weighted images, the fluid in the tumor showed highintensity, suggesting the accumulation of old blood or fluid with high protein concentration (Fig. 2a, b). In addition, there were small mural nodules showing contrast enhancement in the posterior wall of the cystic tumor (Fig. 2c). Based on these observations, the initial impression was that the tumor was a malignant right ovarian tumor, probably clear cell carcinoma arising from an endometriotic cyst. Alternatively, the multilocular nature of the tumor suggested the ovarian tumor to be borderline or malignant mucinous neoplasm.

We also observed an accumulation of large and small cysts in the higher region of the uterine cervix (Fig. 2e, f). There were relatively large cysts in the periphery and small cysts in the central region showing the "cosmos" sign, which is the representative MRI image for LEGH of the uterine cervix, rather than that of MDA.

Dr. Suzuki It is very important for gynecologists to make a differential diagnosis between MDA and LEGH. MDA or "adenoma malignum" is a rare malignant tumor of the cervix [1, 2], whereas the latter is an MDA-related, benign endocervical lesion that was established by Dr. Nucci [3] and Dr. Mikami [4]. It has recently been reported that MDA usually shows a solid pattern rather than a multicystic appearance on the MRI image [5].

Dr. Umeoka (Radiologist) On the abdominal CT scan (Fig. 3), there were many round nodal polyps in the intestines and in the stomach wall (Fig. 3a, b), which suggested that these polyps were associated with PJS. In addition, there was a tumor-like lesion that extended from the left upper quadrant to the mesogastrium (Fig. 3c). Because it looked like the small intestine entered into the small intestine, this was considered to be an invagination. In addition, the CT scan also suggested that invagination occurred at the anal side of the ligament of Treitz, which is a relatively proximal part of the jejunum.

Dr. Miyamoto (Gastroenterology physician) We performed an esophagogastroduodenoscopy (EGD) (Fig. 4). According to the EGD, there were no polyps in the esophagus, but a number of semi-pedunculated polyps (the largest was $20 \mathrm{~mm}$ in diameter) from the stomach (Fig. 4a) to the descending limb of the duodenum (Fig. 4b). According to the colonoscopy, there were also a number of pedunculated polyps (the largest was $20 \mathrm{~mm}$ in diameter) 

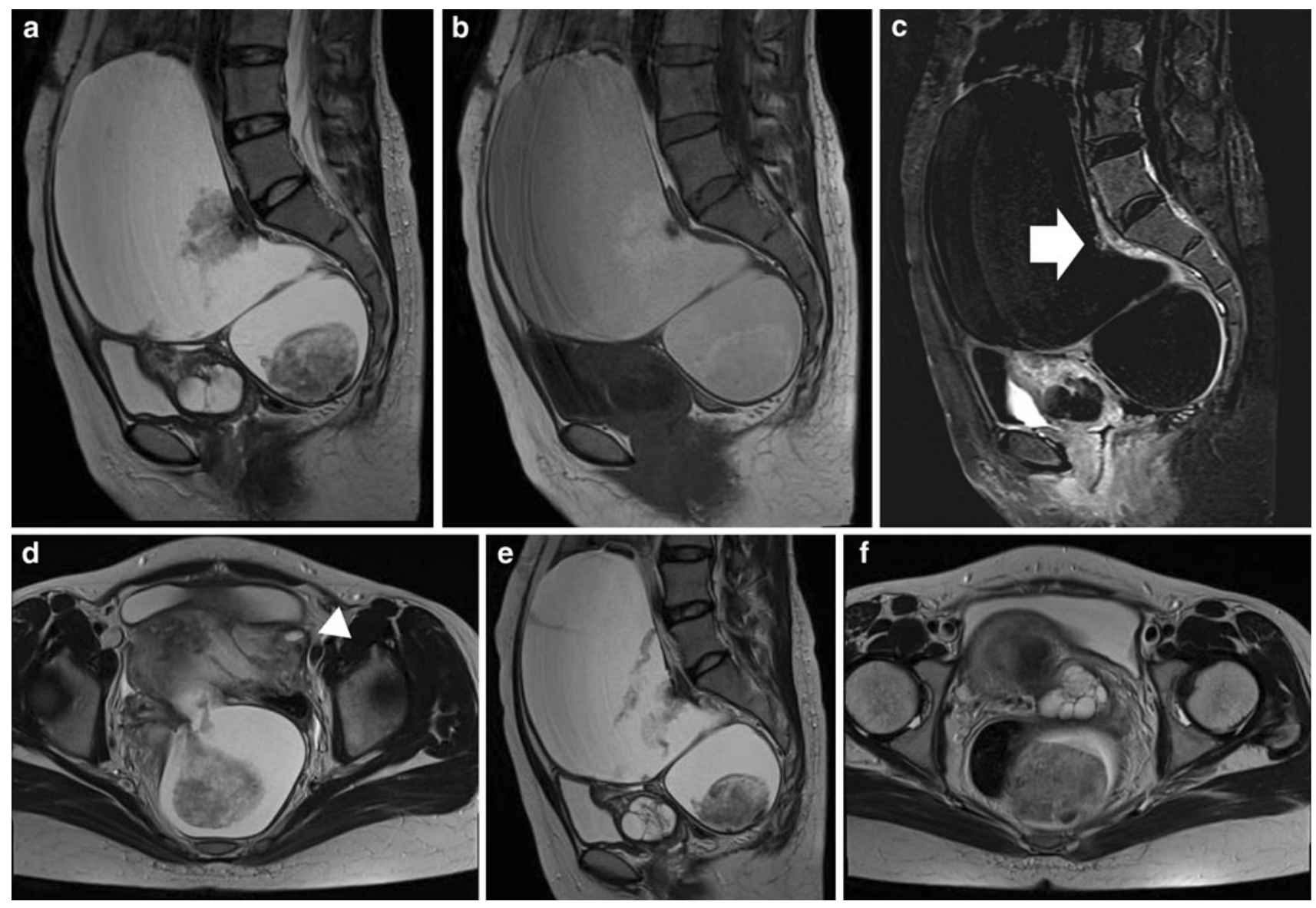

Fig. 2 MRI images of the pelvic area. a T2-weighted image of the ovarian lesion, b T1-weighted image of the ovarian lesion, $\mathbf{c}$ subtraction image of the ovarian lesion, $\mathbf{d}$ T2-weighted image of the left ovary, e T2-weighted image of the uterine cervix, $\mathbf{f}$ T2-weighted image of the uterine cervix. In the pelvis, there was a $22 \times 16-\mathrm{cm}$ bilocular cystic tumor with a clear boundary that was thought to originate from the right ovary. The intratumor fluid was hyperintense in both T1- and T2-weighted images $(\mathbf{a}, \mathbf{b})$. There were mural nodules associated with contrast enhancement in the posterior wall (c arrow). The left, normal-appearing ovary can be seen on the left dorsal to bladder (d arrowhead). In the uterine cervix, there was an accumulation of large and small cysts. There were relatively larger cysts in the periphery and small cysts centrally, referred to as the "cosmos sign" $(\mathbf{e}, \mathbf{f})$ distributed from the rectum to the ascending colon (Fig. 4c). We performed a biopsy of the polyps from the stomach, duodenum, descending colon, and rectum. All of them were diagnosed as hamartomatous polyps of PJS with no evidence of malignancy.

We also investigated the small intestine (Fig. 5). Double contrast study of the small intestine showed that the distal side of the ligament of Treitz exhibited a narrowing (Fig. 5a), consistent with a tightening effect. Interestingly, there was a large tumor in the lumen just above the narrow segment (Fig. 5b), but the passage of contrast media was maintained (Fig. $5 \mathrm{c}$ ). The currently available method to observe the small intestine is either capsule endoscopy or double-balloon endoscopy (Fig. 6). In the present case, peroral double-balloon endoscopy revealed that the massive tumor in the distal side of the ligament of Treitz consisted of an accumulation of large polyps (Fig. 6c). In addition, there were also many polyps of $10-20 \mathrm{~mm}$ in diameter in other regions of the jejunum (Fig. 6a, b, d).

Since there was no problem with the passage through the intestine during endoscopy, I had an impression that invagination had not occurred. However, the CT scan demonstrated invagination, and its treatment is to remove the polyps. Because it is difficult to resect such large polyps endoscopically, the treatment ended up being intervention by surgeons.

Dr. Yasumoto With regard to the nodules in the right breast, ultrasonography revealed the presence of a solid tumor of 12-mm diameter with an indistinct boundary in the right A region (Fig. 7a) and of another tumor of $10 \mathrm{~mm}$ in diameter with a distinct boundary in the right AC region (Fig. 7b). Although the MRI image did not exclude the possibility of malignancy in the A region, we 

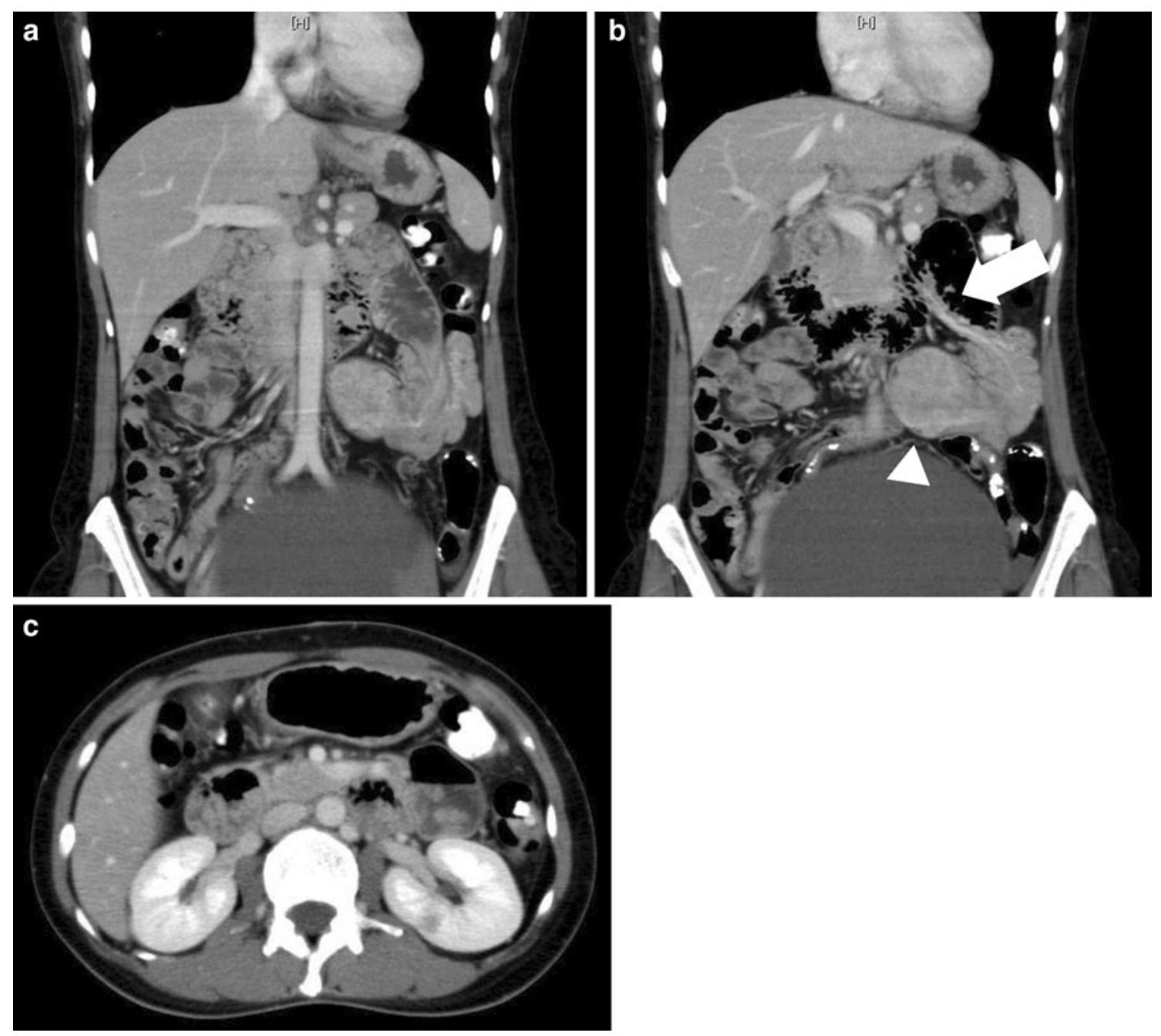

Fig. 3 CT image of the upper quadrant. a, b There were many round nodal polyps in the intestine and stomach walls. $\mathbf{c}$ There was a tumor lesion in the left upper quadrant. The mesenteric vasculature was involved with the tumor as the leading part, and invagination was suggested

prioritized treatment of the digestive tract and gynecological lesions.

\section{Clinical diagnosis}

1. Peutz-Jeghers syndrome

2. Right ovarian tumor: s/o mucinous borderline tumor or clear cell carcinoma in endometriotic cyst

3. Cystic lesions of the uterine cervix: s/o LEGH

4. Digestive tract polyposis and invagination

5. Right breast tumors

Dr. Suzuki Are there any questions concerning the clinical findings, image finings, data on laboratory examination, and clinical diagnosis for this patient?

Dr. Konishi (Gynecologist) After the gynecological examination, did you diagnose the right ovarian tumor as clear cell carcinoma in an endometriotic cyst? The cystic tumor is too large to consider the endometriotic cyst. And, I first considered the possibility of metastatic mucinous ovarian tumor from cervical MDA, because it is known that MDA frequently occurs in PJS patients. It is clinically important that when cervical and ovarian mucinous carcinomas are simultaneously found, the ovarian lesion is usually metastatic from cervical adenocarcinoma.

Dr. Yasumoto From a gynecological point of view, we initially thought that the tumor was a mucinous cystic tumor by its multilocular nature. On the other hand, MRI findings showed the presence of old blood in the tumor associated in part with contrast enhancement. Thus, the radiologists' impression was clear cell carcinoma, which frequently arises in ovarian endometriotic cysts. Regarding the cervical lesion, the preoperative diagnosis was LEGH, since the cervical cytology was negative, and there were no solid lesions on the MRI images. 

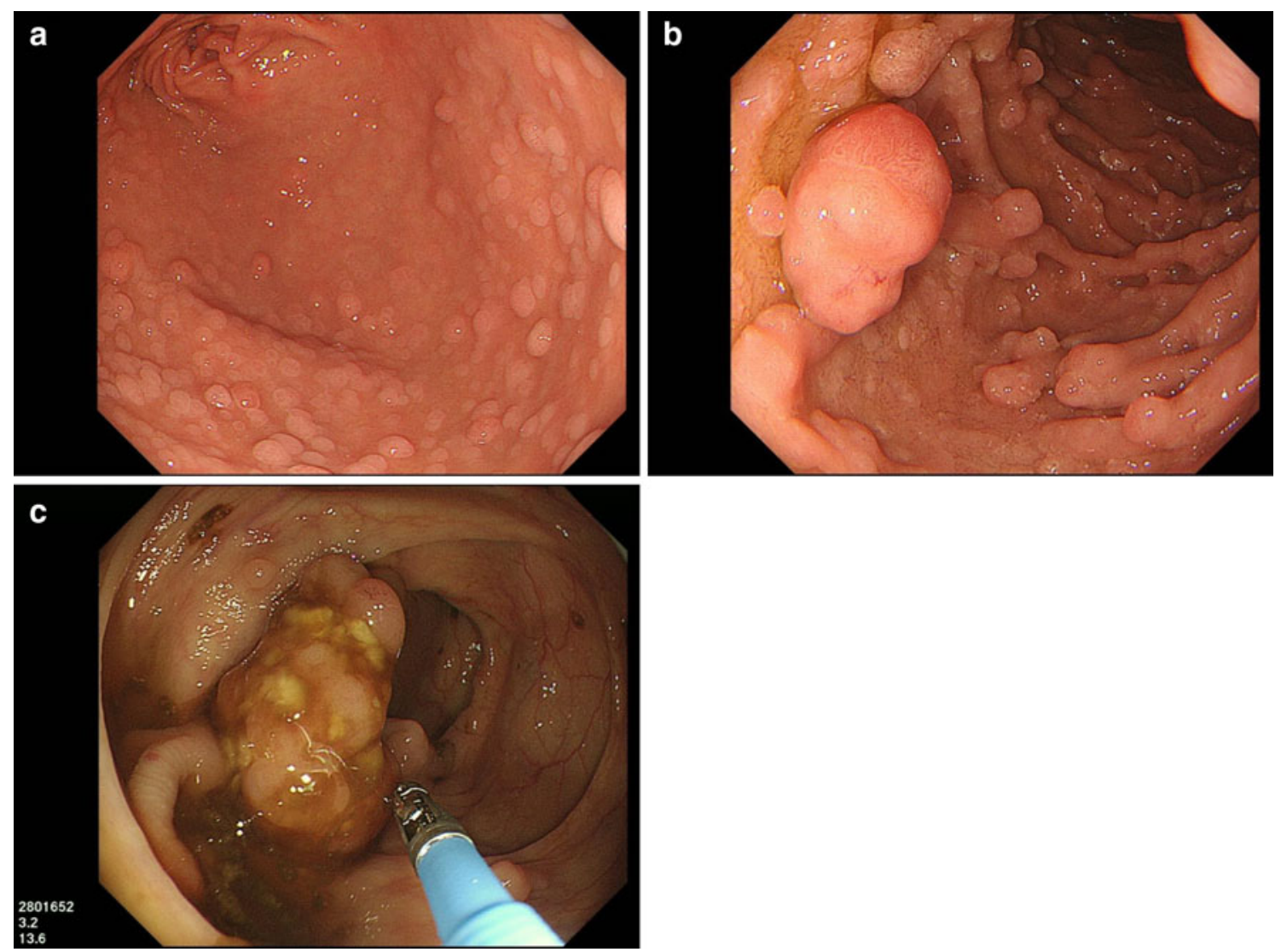

Fig. 4 Endoscopic findings of the upper and lower digestive tract. Pedunculated and semi-pedunculated polyps existed in the stomach (a), duodenum (b), and colon (c)

Dr. Minamiguchi (Pathologist) Is the gastric mucus detection test using HIK1083 performed frequently in gynecological practice?

Dr. Suzuki Yes. A kit is currently available for detecting gastric mucus in the cervical mucus (Kanto Chemical Co., Inc.). We actively use this kit in patients who are suspected of having cervical glandular lesions of gastric phenotype, i.e., when prominent watery discharge is found in the speculum examination or when multiple cystic lesions in the cervix are detected by transvaginal US. The diagnostic efficiency for detection of LEGH and MDA is reported to be relatively good $[5,6]$.

Dr. Obama (Surgeon) I have a question to Dr. Miyamoto. During endoscopy, did you notice invagination?

Dr. Miyamoto No, there were no endoscopy findings indicating invagination. When invagination occurs, patients usually suffer from illness. Strikingly, the patient did not show any subjective symptoms suggesting invagination, such as stomach ache and stool abnormality.

Dr. Matsuura (Gastroenterology physician) In addition, the operability of the digestive tract endoscopy was very good. Using intestinal fluoroscopy, we also confirmed that the contrast media flowed into the small intestine, and we did not expect invagination.

\section{Treatment plans and informed consent}

Dr. Yasumoto Based on the clinical diagnoses, we discussed the choice of treatment with the patient and her family. The patient was nulligravid, and wished the fertility-sparing surgery preserving her uterus and ovaries.

Regarding the right ovarian tumor, we suspected a mucinous cystic tumor of at least borderline malignancy and also could not exclude the possibility of clear cell carcinoma according to the image diagnosis. Thus, we recommended our plan to initially perform right salpingooophorectomy and conduct intraoperative pathology using frozen sections to the patient. If borderline malignant, we would preserve the left ovary, and if fully malignant, we would perform hysterectomy, left salpingo-oophorectomy, and appendectomy.

Regarding the cystic lesion of the uterine cervix, which was strongly suspected to be LEGH, we recommended hysterectomy to the patient. It has recently been reported that approximately $10 \%$ of cases diagnosed as LEGH by 

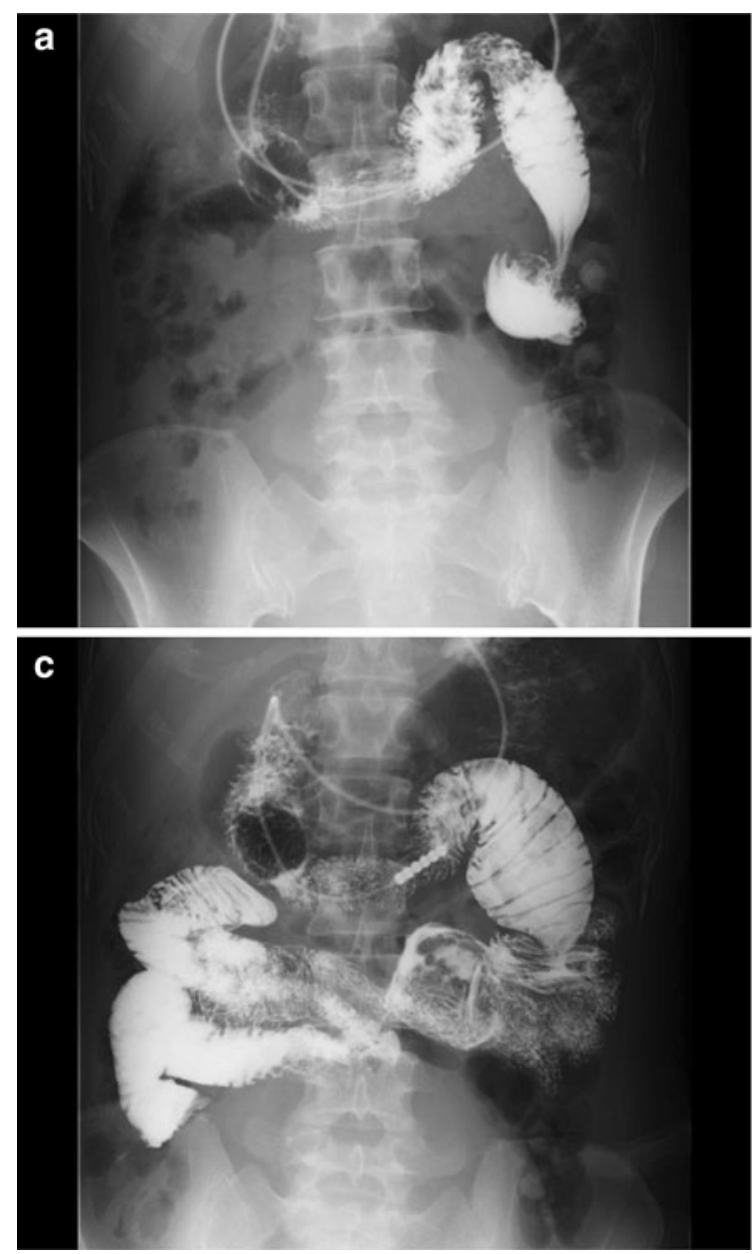

Fig. 5 Double contrast study of the small intestine. a In the distal side of the ligament of Treitz, there was a narrow image that reflected a tightening from the surrounding region. b Right over the narrow

MRI have co-existent adenocarcinoma in situ (AIS), MDA, or invasive mucinous adenocarcinoma [5]. Also, because the lesion was on the higher part of the cervix, it would be difficult to examine the entire lesion by conization. The patient ultimately agreed to the hysterectomy.

Regarding the polyps in the digestive tract, we decided to perform an intestinal resection at the same time as the gynecological surgery, because it is impossible to remove large polyps by endoscopy. The range of intestinal resection would be based on the intraoperative observation.

Regarding the breast nodules, we decided not to do surgery but to follow up after the gynecological and intestinal surgeries. However, since women with PJS have a higher risk of breast cancer, she should undergo cytological and histological examination of the breast lesions in the near future.
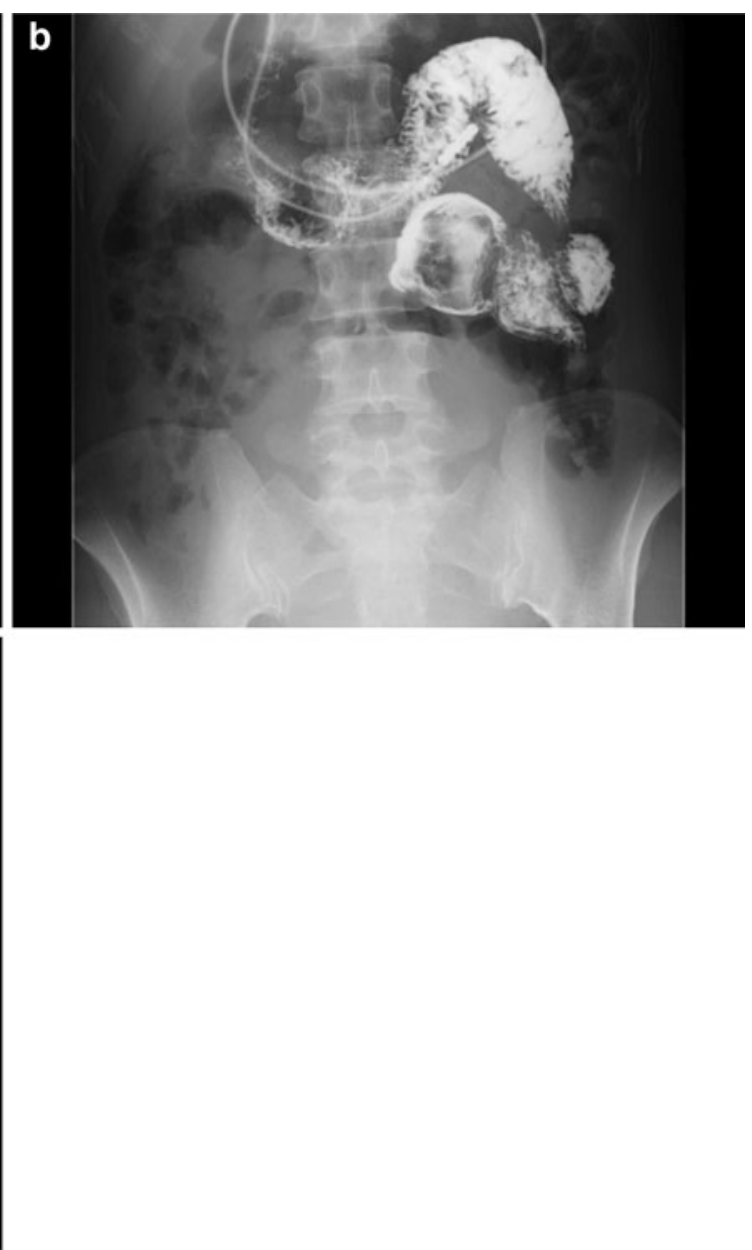
segment, there was a large tumor occupying the lumen. c There were
also many polyps in the ascending limb of the duodenum. Passage of the contrast media was maintained

\section{Operative findings and postoperative course}

Dr. Yasumoto Laparotomy revealed the presence of a small amount of ascites, which were cytologically negative for malignant cells. The pelvic tumor was a huge cystic tumor of the right ovary, whereas the left adnexa were normal. The uterine corpus was normal in size, but the cervix was enlarged. The posterior surface of the uterus was adherent to the rectum, which was caused by endometriosis. First, we performed right salpingo-oophorectomy for the right ovarian tumor, which contained brown-colored fluid. We presented pathological examination of this tumor, including of the solid-like part. Unexpectedly, no malignancy was found in the right ovarian tumor, and therefore we decided to preserve the left ovary, but performed left salpingectomy. Then we performed a hysterectomy, and in the resected specimen, a large amount of mucus leaked from the cervix. 

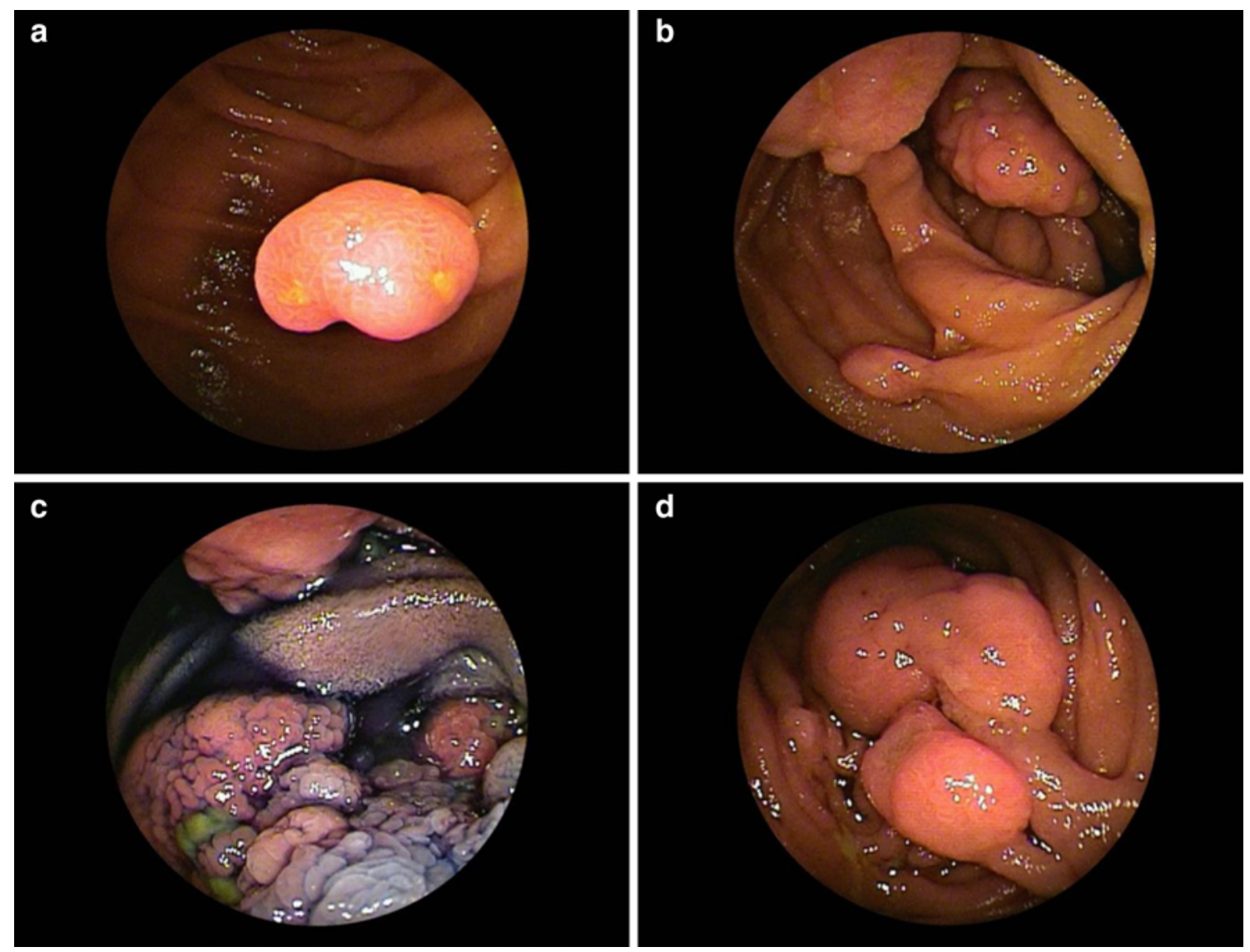

Fig. 6 Double-balloon endoscopic findings. There were many pedunculated polyps in the jejunum (a, b, d). The lesion in the distal side of the ligament of Treitz was composed of multiple polyps (c)
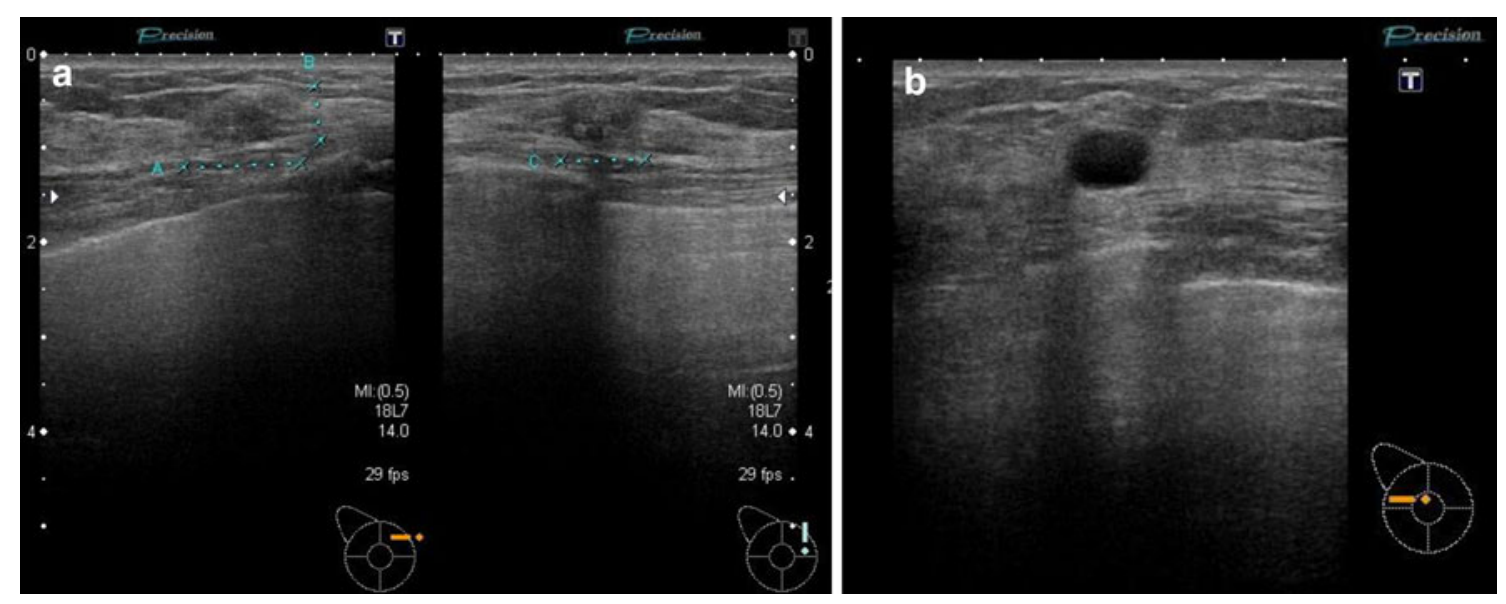

Fig. 7 Mammary ultrasonography. a Right A region: there was a 12-mm tumor with an indistinct boundary. b Right AC region: there was a 10 -mm tumor with a distinct boundary

Dr. Obama I will present the operative findings of the digestive tract. There was a very large palpable tumor on the anal side $20 \mathrm{~cm}$ from the ligament of Treitz. The tumor formed an invagination of this region of the intestine, and therefore we decided to remove the region with the tumor.
The critical point was determining how much we should preserve the small intestine, which still harbored small polyps. Because resection of the entire small intestines causes short bowel syndrome, we preserved the intestines that could be observed by endoscopy. Thus, we removed 

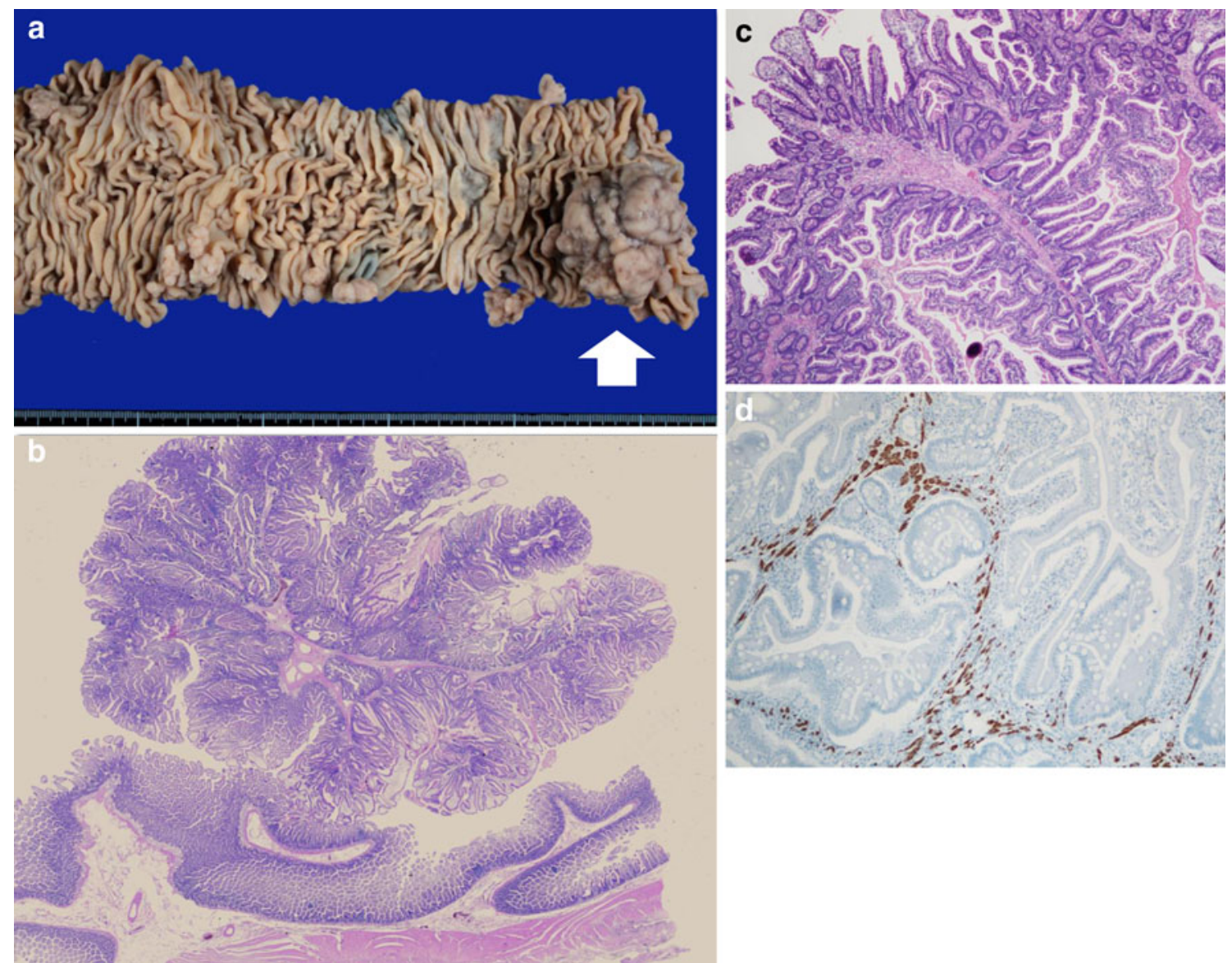

Fig. 8 Observation of the jejunum macroscopically and histopathologically. a Macroscopy of the dissected jejunum, b, c histopathological image of a polyp (H\&E), $\mathbf{d}$ histopathological image of a polyp (desmin), e macroscopic image of a very large polyp, $\mathbf{f}-\mathbf{j}$ histopathological image of a very large polyp (f H\&E, g p53, h Ki-67, i HIK1083, j MUC6). There was a $5 \times 5$-cm tumor (very large polyp) $1 \mathrm{~cm}$ from the proximal stump of the jejunum (arrow). In addition, there were large and small, pedunculated and semi-pedunculated polyps from the distal to the proximal region, which were thought to

$40-50 \mathrm{~cm}$ of the jejunum from the huge polyps of the leading part of the invagination and then performed functional end-to-end anastomosis.

Dr. Yasumoto Accordingly, the performed surgeries were hysterectomy, right salpingo-oophorectomy, left salpingectomy, and partial resection of the jejunum. The postoperative course was almost uneventful, except for mild symptoms of subileus on the 6th postoperative day. However, the passage was fairly good, and the patient was discharged on the 25 th day.

\section{Pathological findings}

Dr. Mikami (Pathologist) Now, you can see the macroscopy of the resected jejunum (Fig. 8). The jejunum was be hamartomatous polyps. In a very large polyp, some ducts tended to aggregate with papillary growth that consisted of highly cylindrical atypical cells. The nuclei were of various sizes and shapes, and invagination was present, being suggestive of adenocarcinoma. However, there was no obvious stromal invasion. Thus, our diagnosis was intraepithelial carcinoma restricted to the mucosal layer. Importantly, the tumor was positive for Ki-67, p53, HIK1083, and MUC6

$42 \mathrm{~cm}$ long, and there was a $5 \times 5 \mathrm{~cm}$ tumor (huge polyp) on the region $1 \mathrm{~cm}$ from the proximal stump. In addition, there were numerous large or small, pedunculated or semipedunculated polyps from the distal to the proximal region. Microscopically, theses polyps had a fine interstitial core and consisted of mucosal tissue from the normal intestinal epithelium (Fig. 8b, c). Immunostaining showed that the interstitial region was positive for desmin, a smooth muscle marker (Fig. 8d). Thus, these polyps were diagnosed as a characteristic hamartomatous polyp of PJS, where smooth muscle bundle formation is associated with dendritic branches in the interstitial region. In the jejunum region, the intrinsic muscle was elevated to the lumen side, forming a "pseudoinvasion."

Next, I will present the cut section (Fig. 8e) and histology of the huge polyp (Fig. 8f-j). Basically, these were hamartomatous polyps; however, some ducts tended to 

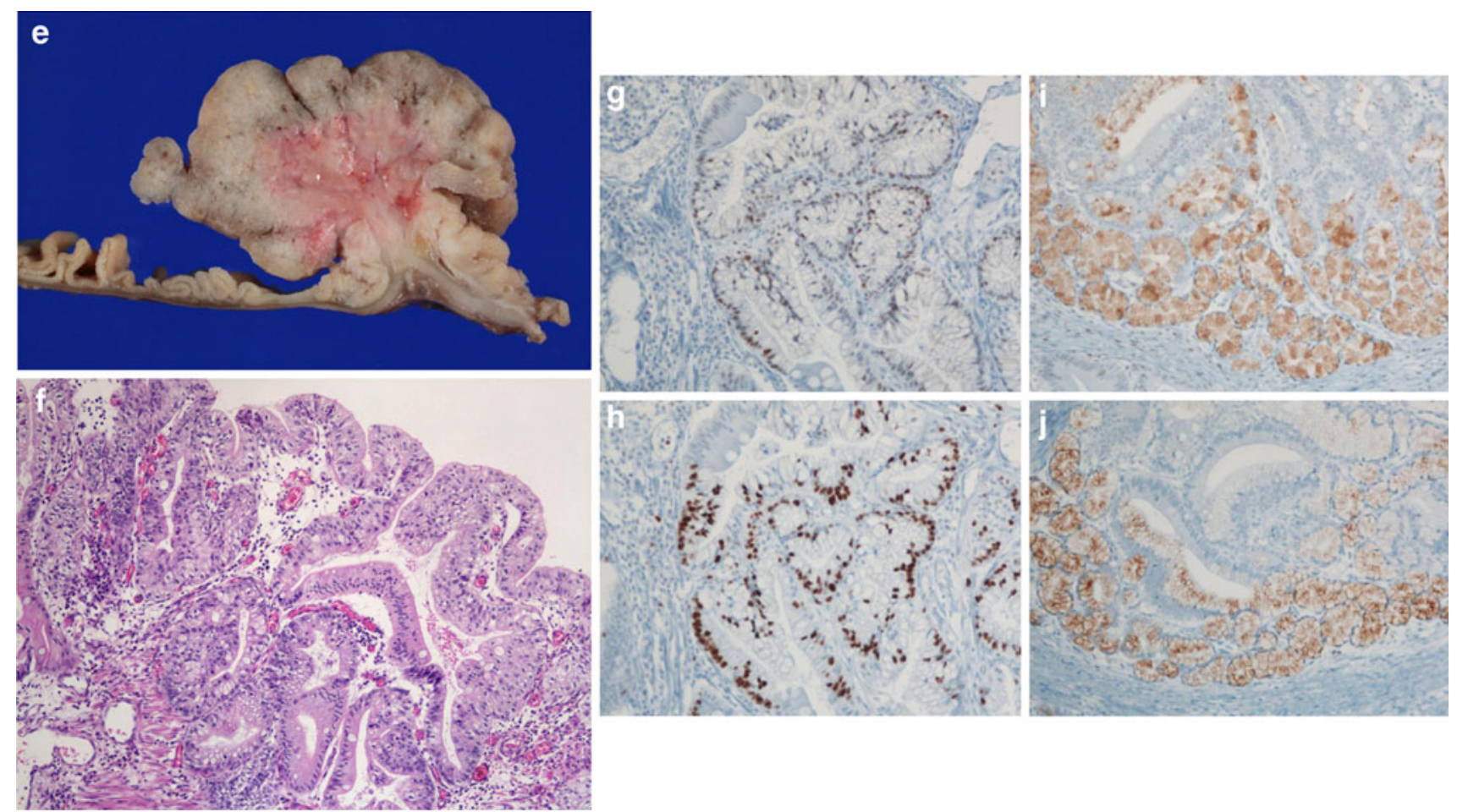

Fig. 8 continued

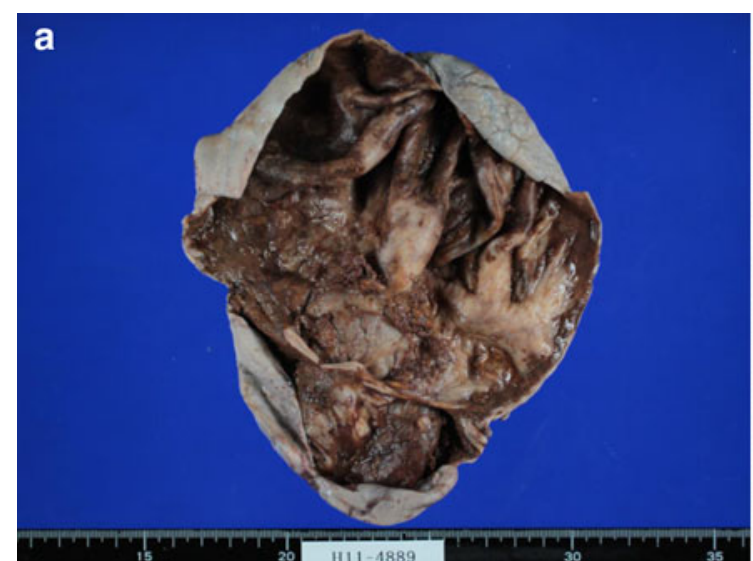

Fig. 9 Macroscopy of the right adnexa and the histopathological findings. a Macroscopic findings, b histopathological image. The lumen was brown in color and was macroscopically suggestive of an

aggregate with papillary growth that consisted of tall columnar atypical cells (Fig. 8f). The nuclei had different sizes and shapes, and showed high levels of invagination, being strongly suggestive of adenocarcinoma. However, there was no obvious stromal invasion, and therefore we concluded that these were intraepithelial carcinomas that were restricted to the mucosal layer. In this atypical lesion, immunohistochemistry confirmed an increase in $\mathrm{Ki}-67-$ positive (Fig. 8g) and p53-positive (Fig. 8h) cells. These atypical cells also showed gastric-type characteristics, as

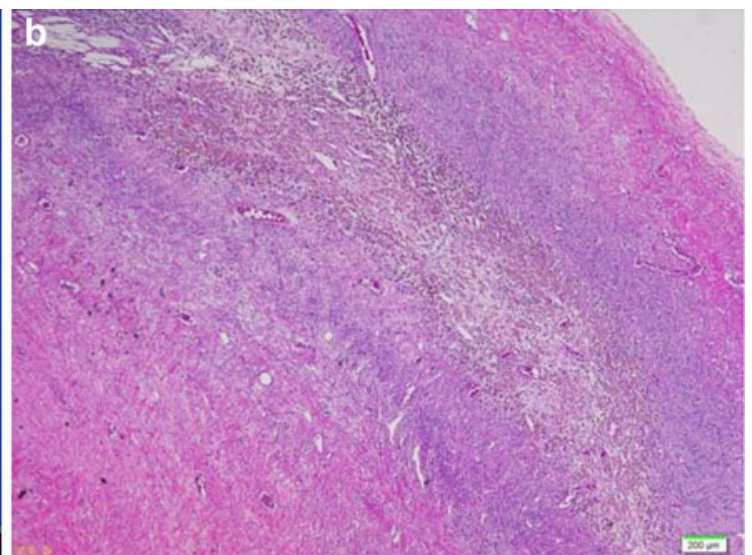

endometriotic cyst. The cyst was thick, but the solid part was not identified. The formation of granulation tissue and fibrosis in the wall was not malignant, but consistent with that of endometriotic cyst

shown in the positivity for HIK1083 (Fig. 8i) and MUC6 (Fig. 8j).

Regarding the right ovary, the lumen was brown in color and was macroscopically suggestive of an endometriotic cyst (Fig. 9a). The cyst was thick, but the solid part was not identified. We carefully analyzed the hyperplastic portion, but no neoplastic or malignant changes were observed in the endometriotic cyst (Fig. 9b).

Regarding the uterus, the upper part of cervix was swollen (Fig. 10a), and there were numerous $1.5-2-\mathrm{cm}-$ 

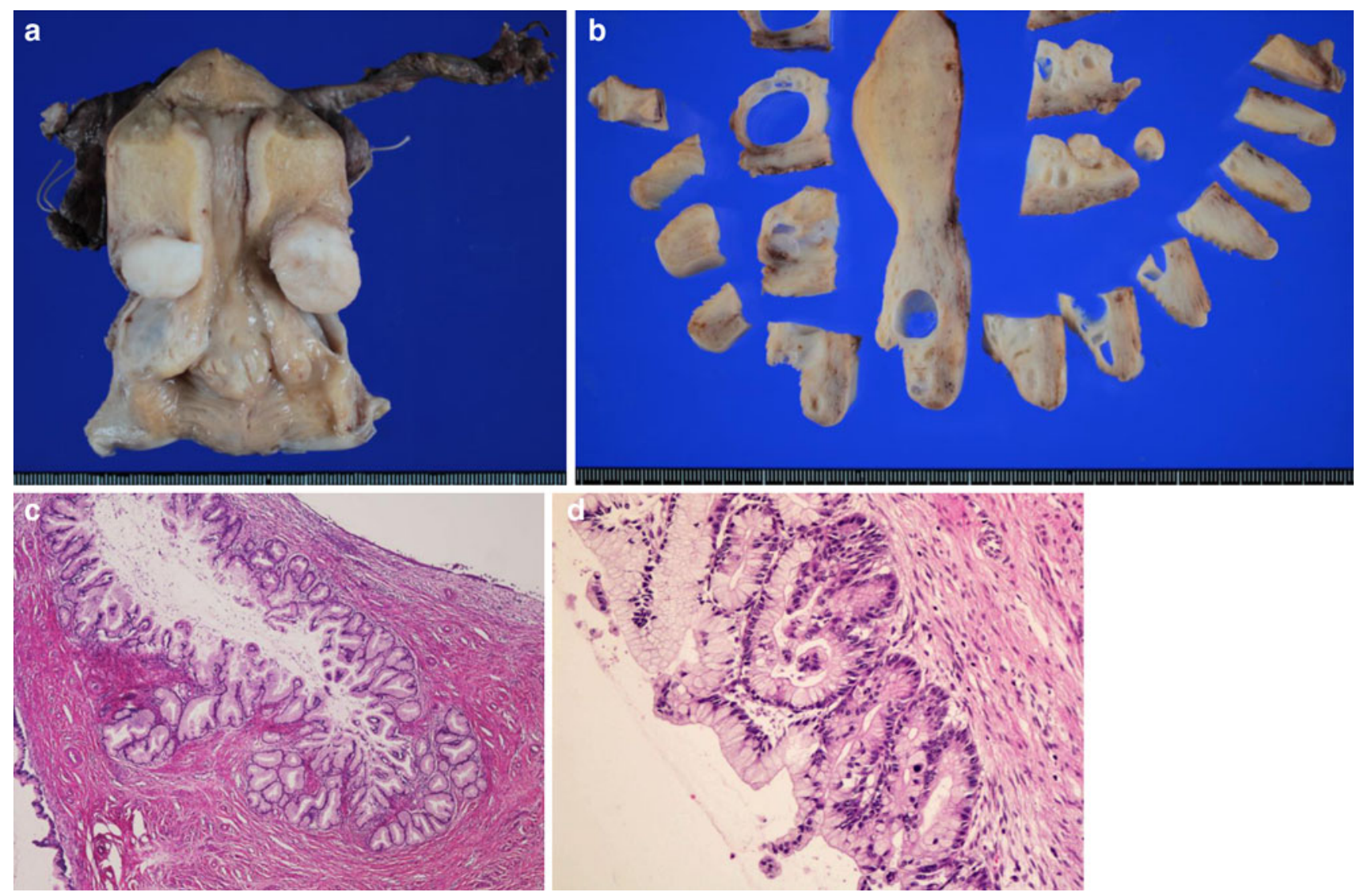

Fig. 10 Macroscopy of the uterus and the histopathological findings of the cervix. a, b Macroscopic findings, $\mathbf{c}, \mathbf{d}$ histopathological image. The upper part of the cervix was swollen (a), and there were numerous 1.5-2-cm-sized cysts (b). The histology of this region showed small ducts and clusters of non-atypical endocervical

sized cysts (Fig. 10b). The histology of this multicystic lesion showed small ducts and clusters of non-atypical endocervical glandular cells, with their prominent lobular structure, which were diagnosed as LEGH (Fig. 10c). Immunohistochemistry also revealed the lesion to be positive for HIK1083 and MUC6, and negative for p53. In addition, one area consisted of moderately atypical glandular cells with nuclear atypia, which might represent early malignant change, being compatible with the diagnosis of atypical LEGH (Fig. 10d). There were no invasive features accompanied by the stromal desmoplastic reaction.

Interestingly, the right fallopian tube had a cystic legion near the fimbria (Fig. 11a) composed of accumulation of gastric-type small ducts resembling those of LEGH (Fig. 11b) and was positive for HIK1083 (Fig. 11d) and MUC6 (Fig. 11e). In addition, a region in the center of the right tube had a lining epithelium that was completely replaced by gastric-type cells without atypia (Fig. 11c). The left oviduct was normal. glandular cells, with their prominent lobular structure (c), which were diagnosed as typical LEGH. One area consisted of moderately atypical glandular cells with nuclear atypia, consistent with the diagnosis of atypical LEGH (d)

\section{Final pathological diagnosis}

1. Endometriotic cyst of the right ovary, no malignancy

2. LEGH with focal nuclear atypia ("atypical LEGH") of the uterine cervix

3. Mucinous metaplasia (gastric metaplasia) of the right fallopian tube

4. Hamartomatous polyps (Peutz-Jeghers type)

5. Adenocarcinoma in Peutz-Jeghers polyp with pseudoinvasion into muscularis propria, $\mathrm{pM} / \mathrm{pTis}$, of the jejunum

\section{Discussion on cancer risk in PJS patients}

Dr. Suzuki First, I would like to ask Dr. Yasumoto to introduce PJS, and the cancer risk and surveillance in PJS patients.

Dr. Yasumoto PJS is first recognized by the frequent occurrence of hamartomatous polyps in the digestive tract 

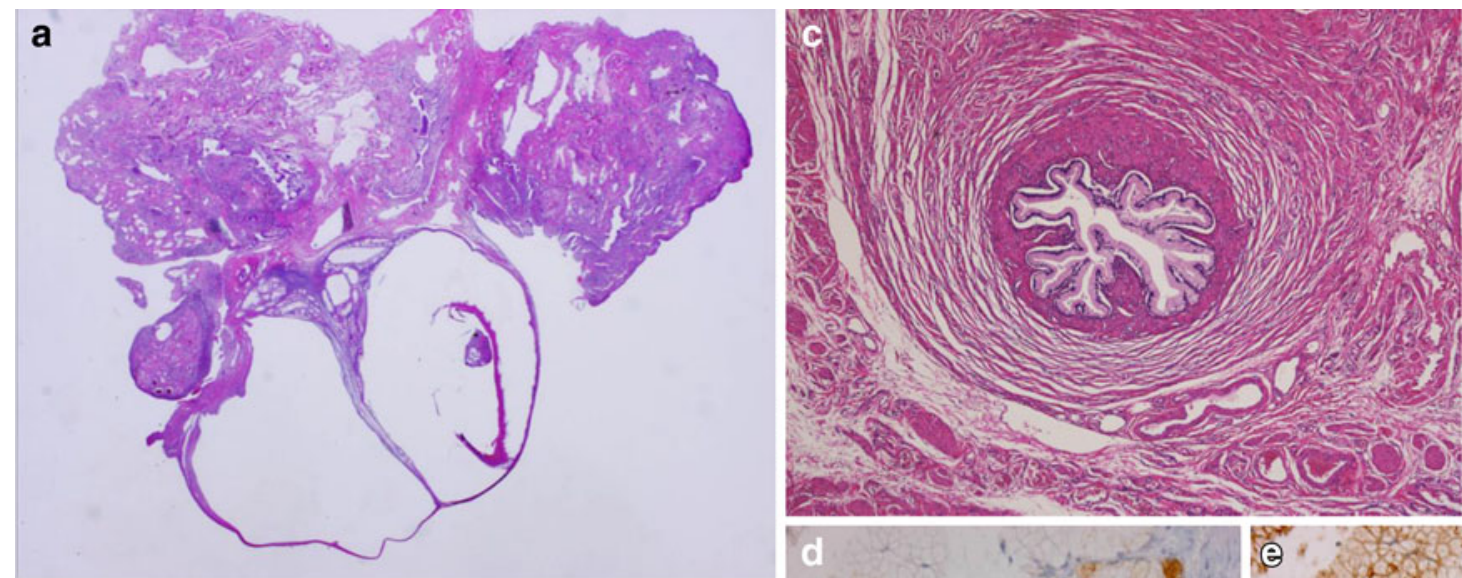

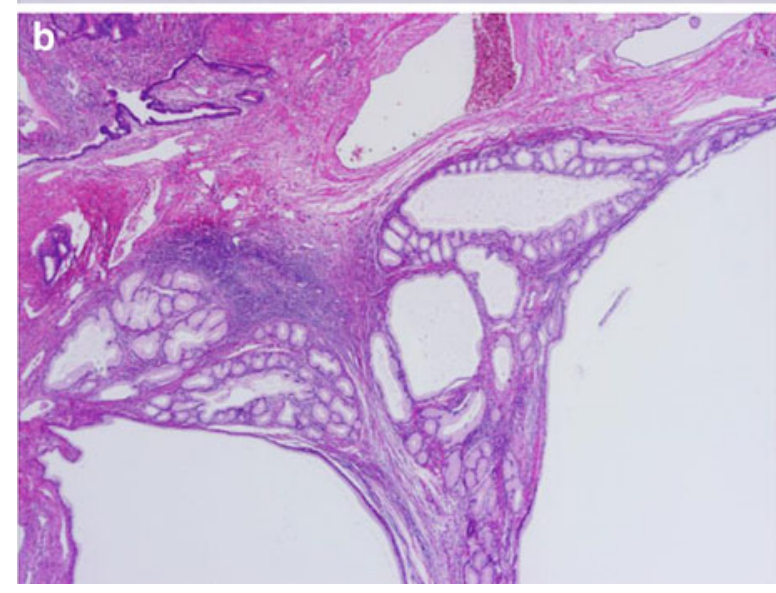

Fig. 11 Histopathological findings of the right oviduct. A cystshaped region near the fimbria of the right fallopian tube (a) was composed of an accumulation of gastric-type small ducts resembling

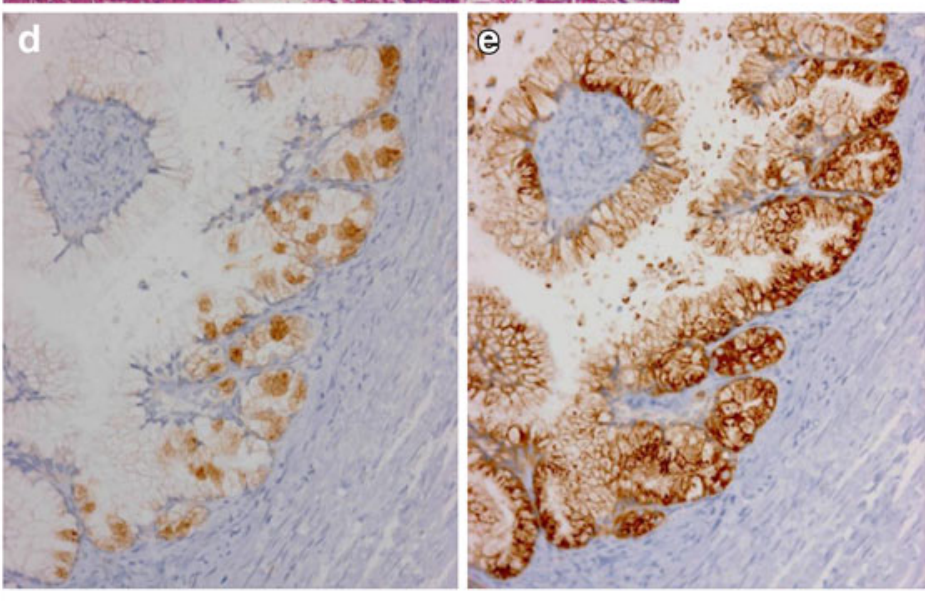

those of LEGH (b). In the right oviduct, a region was completely replaced by gastric-type cells without atypia (c). The cyst-shaped region was positive for HIK1083 and MUC6 (d, e)

Table 1 Cancer risk and surveillance guidelines for Peutz-Jeghers syndrome (cited from the NCCN Guidelines ${ }^{\mathrm{TM}}$ Version 2.2011 Colorectal Cancer Screening)

\begin{tabular}{|c|c|c|}
\hline Site (\% risk) & Screening procedure and interval & $\begin{array}{l}\text { Initiation age } \\
\text { (years) }\end{array}$ \\
\hline Breast $(45-50)$ & $\begin{array}{l}\text { Mammogram and breast MRI annually } \\
\text { Clinical breast exam every } 6 \text { months }\end{array}$ & $\sim 25$ \\
\hline Colon (39) & Colonoscopy every $2-3$ years & $\sim$ Late teens \\
\hline Stomach (29) & Upper endoscopy every $2-3$ years & $\sim$ Late teens \\
\hline Pancreas $(11-36)$ & $\begin{array}{l}\text { Magnetic resonance cholangiopancreatography and/or endoscopic ultrasound every } 1-2 \text { years } \\
\text { CA19-9 at similar intervals }\end{array}$ & $\sim 25-30$ \\
\hline Small intestine (13) & $\begin{array}{l}\text { Small bowel visualization (CT enterography, small bowel enterolysis) baseline at } 8-10 \text { years with } \\
\text { follow-up interval based on findings but at least by age } 18 \text {, then every } 2-3 \text { years though this may } \\
\text { be individualized, or with symptoms }\end{array}$ & $\sim 8-10$ \\
\hline $\begin{array}{l}\text { Ovary }(18-21) \text {, cervix } \\
(10) \text {, uterus }(9)\end{array}$ & $\begin{array}{l}\text { Pelvic examination and PAP smear annually } \\
\text { Consider transvaginal ultrasound }\end{array}$ & $\sim 18-20$ \\
\hline Testes & Annual testicular exam and observation for feminizing changes & $\sim 10$ \\
\hline Lung (15-17) & $\begin{array}{l}\text { Provide education about symptoms and smoking cessation } \\
\text { No other specific recommendations have been made }\end{array}$ & \\
\hline
\end{tabular}

This guideline is available at http://www.nccn.org/professionals/physician_gls/pdf/colorectal_screening.pdf 
and pigmentation in mucocutaneous regions [7]. PJS is an autosomal dominant disorder, and the expected incidence is 1/25,000-280,000. Germline mutations of the LKB1/ STK11 gene have been identified as the cause of PJS. In addition, PJS is characterized by an elevated risk of cancer development [7, 8] (Table 1). According to a prospective study, the cumulative cancer risk is $76 \%$ at the age of 70 [8]. The risk is prominently elevated in female PJS patients, and common malignancies are gastrointestinal cancers, breast cancer, and gynecological tumors [8].

Among gynecological neoplasms, MDA, which is an extremely well-differentiated adenocarcinoma of the uterine cervix, is common in PJS women [1]. In addition, sex cord tumor with annular tubules (SCTAT) is a well-known ovarian tumor frequently seen in PJS woman [9]. Reports have also indicated the frequent occurrence of ovarian mucinous tumors. Interestingly, the present patient showed multifocal mucinous lesions both in the cervix and oviduct, exhibiting so-called synchronous mucinous metaplasia and neoplasia of the female genital tract (SMMN-FGT).

Thus, it is strongly recommended to conduct cancer screening for PJS individuals. According to the National Comprehensive Cancer Network (NCCN) Guidelines (Table 1) and to other recommendations, PJS patients should have various cancer surveillances with appropriate intervals starting from a younger age.

\section{Discussion on MDA, LEGH, and mucinous metaplasia}

Dr. Suzuki Now, I would like to ask Dr. Mikami to introduce MDA, LEGH, and SMMN-FGT, as well as their possible relationship to PJS.

Dr. Mikami First, I would like to introduce MDA and LEGH. MDA, or "adenoma malignum," has been known as a rare tumor, representing 1-3\% of cervical adenocarcinomas, since Profs. Silverberg and Hart established the entity as an aggressive tumor in 1975 [1, 2]. Recent studies showed that MDA shows a gastric phenotype, and part of the tumor is positive for HIK1083 [6]. MDA has also been known to occur frequently in PJS patients who have the germline mutation of the LKB1/STK11 gene, and interestingly, somatic LKB1/STK11 mutation has been reported in sporadic MDA in non-PJS patients [10].

On the other hand, LEGH is a recently identified benign cervical lesion showing gastric metaplasia and being strongly immunopositive for HIK1083 [4]. Before the recognition of this entity, LEGH had often been regarded clinically and histologically as MDA. The average age of onset is 45 years, and patients with LEGH frequently present with a watery vaginal discharge and tumor formation. We analyzed 1,000 cases of hysterectomy and identified 9 LEGH cases $(0.9 \%)$; therefore, its incidence is much higher than that of MDA.

In addition, during our analysis of LEGH cases, we found that some LEGH cases contained strong atypical cells that might be diagnosed as AIS. Because there are also cases that co-exist with invasive MDA or mucinous adenocarcinoma, LEGH has been thought to be the possible precursor of MDA or other adenocarcinomas [11, 12]. Recent reports have also suggested the common characteristics of MDA and atypical LEGH, i.e., abnormalities in the copy numbers of $1 \mathrm{p}$ and $3 \mathrm{q}$, and a high Ki-67- and p53positivity [13].

SMMN-FGT is an interesting disorder that has received attention [14]. It causes multifocal mucinous lesions in female reproductive organs, such as the ovaries, oviduct, and uterine corpus and cervix. These include mucinous metaplasia, mucinous adenoma, mucinous tumor of borderline malignancy, and AIS or invasive adenocarcinoma, which may occur in different combinations. Although SMMN-FGT is usually sporadic, it has also been reported in PJS patients. In addition, SMMN-FGT shows gastrictype characteristics. Therefore, this case is representative of SMMN-FGT, since the gastric-type mucinous lesions were identified in the cervix and in the fallopian tube. Furthermore, SMMN-FGT lesions frequently occur in women with PJS [15].

Dr. Matsumura (Gynecologist) It is very interesting that LEGH in this case contained an atypical lesion that might represent early malignant change. Thus, this PJS patient had developed multifocal cancer lesions in the intestine and the uterine cervix.

Dr. Baba (Gynecologist) In the present case, there was mucinous metaplasia in the right oviduct but not in the left oviduct. Is this a common feature?

Dr. Mikami Yes, this is common. Some cases show mucinous metaplasia in both oviducts, and other cases show metaplasia in only one oviduct.

Dr. Konishi PJS patients have germline mutations in the LKN1/STK11 gene, which may have a relationship with gastric or pyloric gland metaplasia in various tissues. In this patient, do the polyps in the digestive tract have a gastric phenotype? I would like to know how we can uniformly comprehend the genetic mutations of LKB1/ STK11, mucinous metaplasia, hamartomatous polyps, and tumor development.

Dr. Mikami Basically, hamartomatous polyps are considered to be not neoplastic but polyclonal. Gastric metaplasia, however, occurs at a particular site, and tumorigenesis might occur on the site. In the present case, adenocarcinoma of the jejunum was histologically very similar to the 
pyloric gland epithelium. Thus, the neoplastic transformation from hamartomatous polyps may occur along with pyloric gland metaplasia.

Dr. Yoshioka (Gynecologist) Clinically, can we consider that LEGH in PJS women has a higher risk of tumorigenesis than LEGH in non-PJS women?

Dr. Mikami Since there have been no studies about this issue, I cannot make any conclusions on it.

Dr. Mandai (Gynecologist) PJS women have the germline mutations of the LKB1/STK11 gene and tend to develop MDA. The same mutations are known in sporadic MDA in non-PJS patients [10]. How about in LEGH? Is there any observation about the gene mutations in LEGH in non-PJS women?

Dr. Mikami There has been a study that examined the LKB1/STK11 gene mutation in the tumors diagnosed as MDA [11]. Some of the tumors were from our hospital and actually contained the LEGH. We also confirmed that the gene mutation was present in LEGH. In both MDA and LEGH, therefore, it is likely that the LKB1/STK11 gene mutation has already occurred.

Dr. Miyamoto I have experience with a male patient with PJS. I would like to discuss the cancer risk in male PJS. What kind of tumor is complicated by PJS?

Dr. Minamiguchi Other than gastrointestinal and lung cancers, we should pay attention to the testicular tumor. My experience was a testicular sex cord stromal tumor, which is a hormone-producing tumor. The tumor is identified by the clinical findings of feminization, such as progression of bone age and the formation of female-like breasts. The tumor may cause short stature if it is not removed. Thus, annual examination of the testes has also been recommended.

Dr. Miyamoto Generally, hamartomatous polyps are recognized as benign lesions. It was the first time for us to observe such a large polyp in the digestive tract as well as its malignant change in PJS patients. Therefore, we should be careful if the polyps become very large and shows malignant change. In addition, the large polyp caused the intestinal invagination. Thus, even if there are no clinical symptoms, we should pay attention to the possibility of conditions such as invagination, bleeding, and malignancy. This patient still has many polyps in the small and large intestines, and needs surveillance.

Dr. Kosaka (Gynecologist) In the present case, LEGH and its atypical change is related to PJS. On the other hand, the ovarian tumor was an endometriotic cyst, which has been thought to have no relation to PJS.
Dr. Mikami Yes, it is likely that the patient was incidentally complicated by endometriosis.

Dr. Suzuki Any other questions and discussions? If not, I would like to close this case conference. In summary, the present case is representative of a woman with PJS, who had developed multifocal neoplasms, based on the possible genetic background. In such cases, collaboration among gynecologists, gastroenterologists, radiologists, surgeons, and pathologists was needed to make an adequate preoperative assessment and determine the best management. I thank all of you very much for your kind cooperation.

\section{What we learned from this case conference}

1. When you encounter a woman with PJS, you should assess the cancer risk of the patient.

2. You should refer to the various guidelines for cancer surveillance and management.

3. You should evaluate the intestinal polyps and their clinical complications. Malignant change is possible.

4. For gynecology practice, you should pay attention to the possibility of cervical MDA or LEGH, and ovarian mucinous tumor as well as sex cord tumor with annular tubules.

5. For further understanding, you should determine the LKN1/STK11 gene mutation and simultaneous mucinous metaplasia of the female genital tract.

Addendum After this case conference, the breast tumor in the right A region was diagnosed as carcinoma, with no malignancy in the tumor of the AC region. The patient underwent partial resection of the right breast and biopsy of the sentinel lymph node. Pathological examination of the resected specimen revealed the breast tumor to be invasive ductal carcinoma, scirrhous type, pT1aN0, stage Ia, ER$100 \%$ positive, PgR-100\% positive, HER2-negative, Ki67-13.7\% positive. After the surgery, she was given 50 Gy radiation and antiestrogen treatment with tamoxifen.

Conflict of interest The authors have no conflict of interest to disclose with respect to this manuscript.

\section{References}

1. Silverberg SG, Hart WG (1975) Minimal deviation adenocarcinoma ("adenoma malignum") of the cervix: a reappraisal. Am J Obstet Gynecol 121:971-975

2. Kaku T, Enjoji M (1983) Extremely well-differentiated adenocarcinoma ("adenoma malignum") of the cervix. Int J Gynecol Pathol 2:28-41

3. Nucci MR, Clement PB, Young RH (1999) Lobular endocervical glandular hyperplasia, not otherwise specified: a clinicopathologic analysis of thirteen cases of a distinctive pseudoneoplastic 
lesion and comparison with fourteen cases of adenoma malignum. Am J Surg Pathol 23:886-891

4. Mikami Y, Hata S, Melamed J et al (2001) Lobular endocervical glandular hyperplasia is a metaplastic process with a pyloric gland phenotype. Histopathology 39:364-372

5. Takatsu A, Shiozawa T, Miyamoto T et al (2011) Preoperative differential diagnosis of minimal deviation adenocarcinoma and lobular endocervical glandular hyperplasia of the uterine cervix: a multicenter study of clinicopathology and magnetic resonance imaging findings. Int J Gynecol Cancer 21:1287-1296

6. Ishii K, Kumagaki T, Tozuka M et al (2001) A new diagnostic method for adenoma malignum and related lesions: latex agglutination test with a new monoclonal antibody HIK1083. Clin Chim Acta 312:231-233

7. Beggs AD, Latchford AR, Vasen HFA et al (2010) Peutz-Jeghers syndrome: a systematic review and recommendations for management. Gut 59:975-986

8. van Lier MGF, Westerman AM, Wagner A et al (2011) High cancer risk and increased mortality in patients with Peutz-Jeghers syndrome. Gut 60:141-147

9. Young RH, Welch WR, Dickersin GR et al (1982) Ovarian sex cord tumor with annular tubules: review of 74 cases including 27 with Peutz-Jeghers syndrome and four with adenoma malignum of the cervix. Cancer 50:1384-1402
10. Kuragaki C, Enomoto T, Ueno Y et al (2003) Mutations in the STK11 gene characterize minimal deviation adenocarcinoma of the uterine cervix. Lab Invest 83:35-45

11. Mikami Y, Kiyokawa T, Hata S et al (2004) Gastrointestinal immunophenotype in adenocarcinomas of the uterine cervix and related glandular lesions: a possible link between lobular endocervical glandular hyperplasia/pyloric gland metaplasia and 'adenoma malignum'. Mod Pathol 17:962-972

12. Kawauchi S, Kusuda T, Liu XP et al (2008) Is lobular endocervical glandular hyperplasia a cancerous precursor of minimal deviation adenocarcinoma? A comparative molecular-genetic and immunohistochemical study. Am J Surg Pathol 32:1807-1815

13. Mikami Y, Kojima A, Kiyokawa T et al (2009) Ki67 labeling index and p53 status indicate neoplastic nature of atypical lobular endocervical glandular hyperplasia (ALEGH). Histopathology 55:362-364

14. Mikami Y, Kiyokawa T, Sasajima Y et al (2009) Reappraisal of synchronous and multifocal mucinous lesions of the female genital tract: a close association with gastric metaplasia. Histopathology 54:184-191

15. Kato N, Sugawara M, Maeda K et al (2011) Pyloric gland metaplasia/differentiation in multiple organ systems in a patient with Peutz-Jeghers syndrome. Pathol Int 61:369-372 